\title{
Exactitud diagnóstica de cinco referencias gestacionales para predecir el peso insuficiente al nacer
}

\begin{abstract}
María Victoria Benjumea
Grupo de Investigación Materno Perinatal de Caldas, Departamento de Salud Pública, Universidad de Caldas, Manizales, Colombia.

Introducción. La antropometría materna puede utilizarse para evaluar a la gestante y predecir el peso al nacer.

Objetivos. Determinar la exactitud diagnóstica de cinco referencias gestacionales para predecir el peso insuficiente al nacer.

Materiales y métodos. Estudio longitudinal en 245 gestantes colombianas. Variables: peso al nacer, edad gestacional, edad, peso y estatura maternas. Las referencias antropométricas usadas fueron: ganancia de peso gestacional de Fescina (uruguaya), porcentaje peso/talla e índice de masa corporal de Rosso-Mardones (chilena), índice de masa corporal pregestacional del Instituto de Medicina de Estados Unidos e índice de masa corporal de Atalah y col. (chilena). El peso insuficiente al nacer $(<3.000 \mathrm{~g})$ se definió como prueba de oro. Se calcularon la exactitud diagnóstica, la sensibilidad, la especificidad, los valores predictivos positivo y negativo y la concordancia (índice Kappa) con su correspondiente intervalo de confianza (IC 95\%).

Resultados. La sensibilidad para predecir el peso insuficiente fue mayor en el primer trimestre con las referencias chilenas y, a partir del segundo, con la de Estados Unidos y la de Fescina. La mejor concordancia se presentó entre las tres referencias chilenas y la menor con la de Fescina. A partir del segundo trimestre, la de Atalah y col. alcanzó la mayor exactitud diagnóstica, especificidad y valor predictivo positivo.

Conclusiones. Las referencias antropométricas gestacionales con el comportamiento más disímil fueron las de Estados Unidos y de Fescina. La referencia de Atalah y col. alcanzó la mayor exactitud diagnóstica y podría seleccionarse para predecir el peso insuficiente al nacer.
\end{abstract}

Palabras clave: antropometría, recién nacido de muy bajo peso, índice de masa corporal, sensibilidad y especificidad.

\section{Diagnostic accuracy of five gestational references to predict insufficient birth weight}

Introduction. The maternal anthropometry is a potentially valuable tool in the evaluation of pregnancy status and prediction of birth weight.

Objectives. Diagnostic accuracy of birth weight was compared for five gestational anthropometric references.

Materials and methods. Longitudinal study was designed for 245 pregnant Colombian participants. Variables consisted of birth weight, gestational age, maternal height and weight. The references were as follows: gestational weight gain of Fescina (Uruguayan samples), \% weight/height and body mass index of Rosso-Mardones (Chilean sample), pregestational weight of the Institute of Medicine (United States sample), and the body mass index of Atalah and colleagues (Chilean sample). The insufficient birth weight $(<3,000 \mathrm{~g})$ was defined as the critical threshold value. The following indices, with correspondent confidence intervals $(\mathrm{Cl}$ $95 \%)$ were obtained: diagnostic accuracy, sensitivity, specificity, positive and negative predictive values, and agreement (Kappa index).

Results. The ability to predict the weight $<3,000 \mathrm{~g}$ was higher in the first trimester measurement with the Chilean references, and in the second trimester with the USA and the Uruguayan references. The best concordance was presented between the Chilean references and the 
least with the Uruguayan. After the second trimester, the Chilean reference of Atalah and colleagues provided the best diagnostic accuracy, specificity and positive predictive value.

Conclusion. The gestational anthropometric references that provided the most heterogeneous results were the US Institute of Medicine and the Fescina. Overall, the Atalah reference was the most accurate diagnostic predictor of insufficient weight at birth.

Key words: Anthropometry, infant very low birth weight, body mass index, sensitivity and specificity.

La antropometría materna puede utilizarse para evaluar el estado nutricional de la embarazada o para relacionarla con el crecimiento del feto y predecir el peso del bebé al nacer (1-9). Existen diferencias entre las medidas antropométricas usadas para construir indicadores o proponer metodologías en cuanto a su capacidad evaluativa o predictiva de los fenómenos que se quieren medir en el transcurso y al final del embarazo; esta capacidad depende del tipo de medida antropométrica (tamaño o composición corporal), de su combinación con otras medidas antropométricas, del contexto socioeconómico y cultural en el que se utilicen o apliquen y de los objetivos programáticos de su uso (10-18).

El peso, solo o combinado con la estatura elevada a distintas potencias, se ha considerado en el diseño de las referencias existentes para la atención clínica de las embarazadas. Desde la década de los 40, se han elaborado múltiples gráficas para evaluar el estado nutricional de la embarazada en el continente americano. Las más conocidas en Estados Unidos son la de Chesley, 1944; la de Tompkins y Wiehl, 1951; la de Hytten y Leitch, 1971 (19); la de Gueri y col., $1982(19,20)$; la de Rosso P., 1985 (19,21); la de Brown y col., 1986 (19); la de Husaini, 1986 (19); la de Dimperio, 1988 (19), y la del Instituto de Medicina de Estados Unidos, 1990 (2,22,23).

Las referencias más utilizadas en Latinoamérica han sido la de Arnaldo de Siqueira, 1975 en Brasil $(24,25)$; en el Centro Latinoamericano de Perinatología y Desarrollo Humano de Uruguay

Correspondencia:

María Victoria Benjumea, Carrera 23 \# 75A-05, apto 1B, edificio Las Mercedes, Barrio Milán, Manizales, Caldas. Teléfono 6-8866003, fax: 6-8783060 ext. 31131 benjumear@yahoo.com.ar

Recibido:30/03/06; aceptado: 08/11/06 la de Fescina $\mathrm{RH}, 1983(26,27)$; en Chile, las de Rosso P. y Mardones-SF, quienes primero validaron la curva que Pedro Rosso (21) diseñó en Nueva York con el porcentaje de peso para la talla (\% P/T) (28), y luego, en 1999, elaboraron otra curva con la misma población chilena, pero diseñada con el índice de masa corporal (IMC) gestacional (29). Atalah E. y col. 2001, también en Chile $(30,31)$, diseñaron una curva con el IMC gestacional para reemplazar a nivel nacional el porcentaje de peso para la talla de RossoMardones, la cual fue adoptada por el Ministerio de Salud de Chile a partir de enero de 2005 (32).

La diferencia más notable entre las gráficas diseñadas radica en las metas de aumento de peso para las mujeres con distintos pesos pregestacionales (19). Haas (19) afirmó que la mayoría de las características evaluadas en casi todas las curvas de Estados Unidos "tienen poco fundamento científico que las respalde", pues los promedios de aumento de peso se basan únicamente en datos normativos y no en puntos de corte que representen riesgo de productos al nacer con alteraciones del peso; se carece de información sobre los límites de incremento de peso en las poblaciones estudiadas; no se cuenta con información sobre madres que hayan dado a luz niños enfermos, ni sobre edad gestacional y peso al nacer fuera de lo normal; se elaboraron con gestantes sanas sin complicaciones durante el embarazo, situación que dificulta la definición de límites y puntos de corte apropiados para el incremento de peso de mujeres en situaciones nutricionales extremas con relación a resultados específicos favorables y desfavorables (23), y muchas de las curvas se diseñaron de acuerdo con las necesidades de los clínicos y no para la vigilancia en salud pública o como producto de investigaciones epidemiológicas (19). La conclusión de Haas fue que "es difícil recomendar un patrón específico o un margen de variación en 
los incrementos de peso que se relacionen con resultados gestacionales positivos" (19).

Estos, entre otros, han sido parte de los argumentos de los estudios que se han realizado en Latinoamérica para evaluar la sensibilidad, especificidad y capacidad predictiva negativa y positiva de las referencias disponibles (33-37); por ejemplo, en las curvas de Chile, la mayor diferencia se encontró entre los puntos de corte definidos para cada categoría antropométrica de la gestante $(32,37)$.

En Colombia no se ha diseñado aún una curva o gráfica para evaluar el estado nutricional de la gestante y predecir el peso al nacer del bebé. Se han usado indistintamente las tres referencias chilenas y la del Centro Latinoamericano de Perinatología y Desarrollo Humano de Uruguay para la evaluación del estado nutricional gestacional (38), y no existe una propuesta nacional unificada por el Viceministerio de Salud. Por ello, cada organismo de salud utiliza la que considere pertinente, lo cual ha limitado el análisis nacional y regional de la información antropométrica de las gestantes y la unidad de criterios de tamizaje para la intervención nutricional durante la gestación.

El peso al nacer ha sido usado como prueba de oro en la evaluación de la capacidad predictiva de las diferentes propuestas disponibles para estudiar el estado nutricional de la gestante (3337). La categoría de peso al nacer más comúnmente usada en los estudios referenciados ha sido la de bajo peso (<2.500 g); no obstante, al analizar en Caldas las prevalencias de bajo $(8,0 \%)$ e insuficiente peso al nacer $(23,4 \%)$ (39) y el comportamiento de la morbilidad por bajo $(11,5 \%$, $\mathrm{n}=241)$ e insuficiente peso al nacer $(2.500 \mathrm{~g} \mathrm{a}$ $2.999 \mathrm{~g}: 15,8 \%, \mathrm{n}=331$ ) desde 1998 hasta febrero de 2004, no se encontraron diferencias estadísticamente significativas en la morbilidad (ji : 1,94 , $p=0,163)(40)$.

Con base en esta información y en consideración a la propuesta de Puffer y Serrano (41) sobre la importancia de considerar el peso insuficiente al nacer entre las categorías de riesgo de morbilidad neonatal, se decidió agrupar el peso inferior a 3.000 gramos como peso insuficiente al nacer y usarlo como prueba de oro en este estudio para determinar la exactitud diagnóstica de las referencias antropométricas gestacionales disponibles en América Latina.

\section{Materiales y métodos}

Se llevó a cabo un estudio de tipo longitudinal en las gestantes usuarias del control prenatal de la Asociación de Servicios Básicos de Salud (ASSBASALUD) en el municipio de Manizales, Colombia.

\section{Población de estudio}

Según los criterios de inclusión definidos para este tipo de estudios, se seleccionaron 245 gestantes residentes en la zona urbana del municipio de Manizales, Colombia, que asistieron a su control prenatal entre 1999 y 2000 con embarazo único a término y estaban libres de antecedentes patológicos u obstétricos que afectaran el peso al nacer del bebé.

Los requisitos de inclusión de las gestantes fueron similares a los que consideraron los autores para el diseño de las referencias estudiadas $(20,21,24-$ $26,28,31$ ), y los de exclusión fueron aquéllos que según los autores y la literatura publicada son factores de riesgo de resultados adversos del embarazo $(20,21,24-26,28,31,42-46)$ (cuadro 1).

Las variables del recién nacido fueron el peso y la edad gestacional al nacer, y las maternas fueron la edad cronológica, el peso corporal, la talla y la edad gestacional. El peso insuficiente al nacer se definió como aquel menor de $3.000 \mathrm{~g}$ (incluyó las categorías de bajo, insuficiente o inadecuado peso al nacer); el normal se estableció entre 3.000 y 4.000 gramos y el macrosómico en mayor de 4.000 gramos. Se construyeron los indicadores antropométricos gestacionales de ganancia de peso gestacional: peso actual (kilogramos) - peso previo (kilogramos) y porcentaje de peso para la talla $(26,28)$; índice de masa corporal gestacional: $\mathrm{kg} / \mathrm{m}^{2}(22,29,30,31)$ y pregestacional o hasta la semana 14 de gestación $(22,23)$; gradiente de peso materno: peso actual (gramos) - peso previo (gramos)/edad gestacional actual (semanas) - edad gestacional previa (semanas) y ganancia absoluta de peso materno según el índice de masa corporal pregestacional 
Cuadro 1. Requisitos de inclusión y de exclusión de las gestantes.

\begin{tabular}{|c|c|}
\hline Criterios de inclusión & Criterios de exclusión \\
\hline Residir en el área de cobertura de salud & Período intergenésico $<2$ años \\
\hline Primera evaluación ginecológica y nutricional antes de las 14,6 semanas & Abortos espontáneos previos \\
\hline Edad entre 13 y 40 años (en la muestra colombiana, entre 19 y 35 años) & Antecedentes de infertilidad y de mortinatos \\
\hline $\begin{array}{l}\text { Estudio ultrasonográfico a las } 20 \text { semanas para confirmar edad } \\
\text { gestacional y para detectar posibles malformaciones congénitas } \\
\text { (p.e., defectos del cierre del tubo neural) }\end{array}$ & Embarazo múltiple \\
\hline Paridad entre 0 y 5 & Cirugía ginecológica \\
\hline Parto atendido institucionalmente entre 37 y 42 semanas & $\begin{array}{l}\text { Antecedentes de recién nacidos con bajo } \\
\text { peso al nacer o de fetos macrosómicos }\end{array}$ \\
\hline Recién nacido vivo, sano y único & Prematuridad \\
\hline Contar con medidas antropométricas en los tres controles & $\begin{array}{l}\text { Antecedentes o presencia de malformacio- } \\
\text { nes congénitas e isoinmunización } \mathrm{Rh}\end{array}$ \\
\hline Ausencia de consumo de sustancias psicoactivas, cigarrillo y alcohol & $\begin{array}{l}\text { Antecedentes de salud familiares de } \\
\text { importancia obstétrica y de enfermedad o } \\
\text { patología del embarazo, parto y puerperio }\end{array}$ \\
\hline $\begin{array}{l}\text { Ausencia de anemia, diabetes, hipertensión, cardiopatías, } \\
\text { hemorragias en el embarazo, problemas mentales }\end{array}$ & $\begin{array}{l}\text { Modo de parto: instrumentado (cesárea, } \\
\text { fórceps) }\end{array}$ \\
\hline \multicolumn{2}{|l|}{$\begin{array}{l}\text { Ausencia de problemas genéticos de la madre y de antecedentes } \\
\text { de anomalías congénitas }\end{array}$} \\
\hline Ausencia de infecciones del recién nacido y de patologías obstétricas & \\
\hline
\end{tabular}

$(22,23)$. Personal de salud previamente entrenado, supervisado y con experiencia en la técnica antropométrica obtuvo las medidas antropométricas en cada trimestre; se siguieron las recomendaciones propuestas por Lohman y col. (47).

Todos los procedimientos tuvieron en cuenta las consideraciones bioéticas para la vigilancia epidemiológica de la gestante (48) y las planteadas por el Ministerio de Salud de Colombia (49). Para cumplir con ello, el proyecto se puso a consideración del Comité de Ética de la Facultad de Ciencias para la Salud de la Universidad de Caldas y se le explicó a cada gestante su objetivo para obtener su consentimiento.

\section{Procedimientos}

Las referencias antropométricas para evaluar a la gestante en cada trimestre fueron las cinco disponibles en América Latina:

- ganancia de peso para la edad gestacional de Fescina/CLAP $(26,27,50,51)$;

- porcentaje de peso para la talla según la edad gestacional de Rosso y Mardones $(21,28,29)$;

- índice de masa corporal pregestacional y gradiente de peso materno según este índice trimestral propuesto por el Instituto de Medicina de los Estados Unidos $(2,22,23)$;

- índice de masa corporal gestacional de RossoMardones y col. (29), e

- índice de masa corporal gestacional de Atalah y col. $(30,31,52)$.

La descripción del estado nutricional de las gestantes se realizó con cada referencia utilizando porcentajes para cada categoría antropométrica (normal, bajo peso, sobrepeso o alto y obesidad o muy alto) en el caso de las variables cualitativas, y con la media aritmética, la desviación estándar y los valores mínimo y máximo en el caso de las variables cuantitativas. Para el manejo estadístico de los datos se crearon bases de datos en Excel. Su validez se analizó a partir de la detección de valores extremos mediante la construcción de diagramas de tipo Box-Plot. Los datos extremos encontrados se analizaron y se procedió a eliminar los que eran biológicamente imposibles. Para el procesamiento de la información se utilizaron los programas estadísticos SPSS versión 10.0 y EPIINFO versión 6.04d. Con este último programa 
se calcularon la sensibilidad, la especificidad, los valores predictivos positivo y negativo y los correspondientes intervalos de confianza (IC 95\%).

Para describir la capacidad predictiva de los diferentes criterios de clasificación antropométrica se utilizó el peso insuficiente al nacer como prueba de oro y se calcularon la exactitud diagnóstica (calidad de las referencias antropométricas para tamizar a las gestantes con y sin bebés de peso insuficiente al nacer): VP (verdaderos positivos) $+\mathrm{VN}$ (verdaderos negativos)/ VP + VN + FP (falsos positivos) + FN (falsos negativos) (53), y la sensibilidad, especificidad y valores predictivos positivo -VPP- y negativo -VPN- (54). La evaluación de la concordancia entre criterios de clasificación antropométrica se realizó con las categorías de bajo peso y sin bajo peso gestacional. La concordancia o grado de acuerdo entre los resultados de las referencias antropométricas más allá del azar se caracterizó con el coeficiente kappa y su variabilidad se expresó mediante el error estándar de estimación, a partir del cual se construyó el correspondiente intervalo de confianza (IC 95\%). La interpretación de sus valores se realizó siguiendo la propuesta de Altman DG (55), que plantea las siguientes categorías de concordancia para el índice kappa: pobre, $<0,20$; regular, 0,21 a 0,40; moderada, 0,41 a 0,60; buena, 0,61 a 0,80 y muy buena, 0,81 a 1,00 .

\section{Resultados}

Se evaluaron 245 gestantes con edad promedio de $25,2 \pm 4,6$ años. La edad gestacional del primer trimestre fue en promedio de $11,9 \pm 1,9$ semanas, la del segundo trimestre de $25,9 \pm 1,9$ y la del tercer trimestre correspondió a 37,8 $\pm 1,5$. La ganancia absoluta de peso hasta el último control fue de $10,9 \pm 3,4 \mathrm{Kg}$ y el gradiente de peso entre el primero y último trimestres fue de $369,1 \pm 131,7$ gramos/ semana.

El promedio de peso al nacer de los niños fue de $3.215,0 \pm 369,7 \mathrm{~g}$ (mínimo $2.050 \mathrm{~g}$, máximo $4.800 \mathrm{~g}$ ). Se encontró mayor proporción de peso insuficiente $(<3.000 \mathrm{~g}: 26,1 \%)$ que de bajo peso al nacer (<2.500 g: 0,8\%).

\section{Clasificación antropométrica del estado nutricional gestacional}

En el cuadro 2 se presentan las variables antropométricas maternas en cada trimestre. La ganancia absoluta de peso fue más alta en el segundo trimestre y el gradiente o velocidad de ganancia de peso semanal lo fue entre en el segundo y el tercer trimestres (cuadro 2).

Las referencias de clasificación de la gestante en cada categoría antropométrica fueron diferentes en cada trimestre (cuadro 3 ). Con las dos referencias de Rosso-Mardones se evaluó la mayor

Cuadro 2. Antropometría materna en cada trimestre del embarazo.

\begin{tabular}{lr}
\hline Variable & $\begin{array}{r}\text { Colombia (Manizales) } \\
(\mathbf{n = 2 4 5}) \mathbf{X} \mathbf{( D . S} \text {.) }\end{array}$ \\
\hline Talla materna $(\mathrm{cm})$ & $155,0(5,0)$ \\
Peso materno primer trimestre $(\mathrm{Kg})$ & $56,9(8,3)$ \\
Peso materno segundo trimestre $(\mathrm{Kg})$ & $62,3(8,3)$ \\
Peso materno tercer trimestre $(\mathrm{Kg})$ & $66,7(8,5)$ \\
IMCP $\left(\mathrm{Kg} / \mathrm{m}^{2}\right)$ & $23,6(3,2)$ \\
IMC segundo trimestre $\left(\mathrm{Kg} / \mathrm{m}^{2}\right)$ & $25,9(3,2)$ \\
IMC tercer trimestre $\left(\mathrm{Kg} / \mathrm{m}^{2}\right)$ & $27,7(3,3)$ \\
Ganancia absoluta de peso segundo trimestre - Peso primer trimestre $(\mathrm{Kg})$ & $5,7(2,6)$ \\
Ganancia absoluta de peso tercer trimestre - Peso segundo trimestre $(\mathrm{Kg})$ & $4,4(2,2)$ \\
Ganancia absoluta de peso tercer trimestre - Peso primer trimestre $(\mathrm{kg})$ & $10,1(3,4)$ \\
Gradiente de peso segundo trimestre - Peso primer trimestre $(\mathrm{g} / \mathrm{s})$ & $366,7(170,1)$ \\
Gradiente de peso tercer trimestre - Peso segundo trimestre $(\mathrm{g} / \mathrm{s})$ & $378,9(184,0)$ \\
Gradiente de peso tercer trimestre - Peso primer trimestre $(\mathrm{g} / \mathrm{s})$ & $369,1(131,7)$
\end{tabular}

D.S: desviación estándar

IMCP: índice de masa corporal pregestacional

IMC: índice de masa corporal 
proporción de obesidad durante todo el embarazo, al igual que de bajo peso en el primer trimestre; en el segundo y tercer trimestres, la mayor proporción de bajo peso se evaluó con la referencia del Instituto de Medicina de Estados Unidos.

El sobrepeso fue mayor con la referencia del Centro Latinoamericano de Perinatología y Desarrollo Humano en el primer trimestre, y a partir del segundo trimestre ocupó el último lugar en proporción de gestantes con sobrepeso. La referencia del Instituto de Medicina de Estados Unidos pasó a ocupar el primer lugar en la clasificación de sobrepeso materno (cuadro 3). La mayor normalidad se clasificó con referencias antropométricas diferentes en cada control gestacional así: Instituto de Medicina de Estados Unidos (primer trimestre), Atalah y col. (segundo trimestre) y Centro Latinoamericano de
Perinatología y Desarrollo Humano (tercer trimestre).

El gradiente del peso materno según IMCP y la edad gestacional se presentan en el cuadro 3. Las gestantes que iniciaron su embarazo con IMC bajo o normal ganaron más peso por semana en el segundo trimestre, mientras que en las que lo iniciaron con IMC alto o muy alto, la velocidad de ganancia de peso semanal fue superior en el tercer trimestre (cuadro 4).

\section{Exactitud diagnóstica de las referencias estudiadas}

La exactitud diagnóstica (calidad de las referencias antropométricas para tamizar a las gestantes con y sin bebés de peso insuficiente al nacer) fue superior al $50 \%$ durante el embarazo con casi todas las referencias antropométricas, a

Cuadro 3. Clasificación antropométrica del estado nutricional de las gestantes según referencia antropométrica y edad gestacional.



*Las gráficas evalúan a partir de la semana 10. **La gráfica evalúa a partir de la semana 13. NA: no clasifica obesidad IMC: índice de masa corporal

IMCP: índice de masa corporal pregestacional

IME: Instituto de Medicina de los Estados Unidos

CLAP: Centro Latinoamericano de Perinatología y Desarrollo Humano 
excepción de la referencia del Instituto de Medicina de Estados Unidos (43,0\%, segundo trimestre y $48,0 \%$, tercer trimestre). La exactitud incrementó en $6,0 \%$ al final del embarazo sólo con el IMC de Atalah y col. (cuadro 5).

La mayor pérdida de exactitud diagnóstica se presentó con la referencia del Instituto de Medicina de Estados Unidos (24,0\%) y la menor con el \% P/T (2,0\%) (cuadro 5). La exactitud diagnóstica más alta al final del embarazo se logró con el IMC de Atalah y col; ello se acompañó de un cambio en la clasificación de bajo peso gestacional con respecto al primer trimestre de $2,6 \%$, y en el VPP de $+14,3 \%$ (cuadro 5).

\section{Sensibilidad, especificidad y valores predictivos (negativo y positivo) para peso insuficiente al nacer}

Entre el primer y el tercer trimestres, las referencias antropométricas cambiaron sus propiedades métricas (sensibilidad, especificidad y valores predictivos positivo y negativo). Todas ganaron sensibilidad al final del embarazo (\% P/T de Rosso-Mardones: 10,5\%; IMC de los mismos autores: 17,7\%; IMC de Atalah y col.: 6,2\%; CLAP: $30,4 \%$; Instituto de Medicina de Estados Unidos: 54,6\%), y casi todas perdieron entre 6,6 y $53,1 \%$ de especificidad, excepto la referencia del IMC de Atalah y col. que la incrementó en

Cuadro 4. Gradiente del peso materno según índice de masa corporal pregestacional y edad gestacional.

\begin{tabular}{|c|c|c|c|c|}
\hline \multirow{3}{*}{ Edad gestacional } & \multicolumn{4}{|c|}{$\begin{array}{c}\text { Gradiente del peso materno } \\
\text { X } \pm \text { (D.S.) (gramos/semana gestacional) }\end{array}$} \\
\hline & \multicolumn{4}{|c|}{ Índice de masa corporal pregestacional } \\
\hline & Bajo & Normal & Alto & Muy alto \\
\hline Del primer al segundo trimestre & $422,0 \pm 128,2$ & $379,4 \pm 171,6$ & $321,4 \pm 146,8$ & $263,1 \pm 213,7$ \\
\hline Del segundo al tercer trimestre & $400,9 \pm 179,1$ & $378,5 \pm 185,7$ & $341,0 \pm 155,6$ & $379,4 \pm 171,6$ \\
\hline Del primer al tercer trimestre & $415,7 \pm 114,5$ & $375,2 \pm 136,5$ & $328,5 \pm 95,2$ & $345,3 \pm 164,8$ \\
\hline
\end{tabular}

Cuadro 5. Exactitud diagnóstica de las referencias antropométricas según trimestre.

\begin{tabular}{|c|c|}
\hline Referencia antropométrica & $\begin{array}{c}\text { Exactitud } \\
(\%)\end{array}$ \\
\hline \multicolumn{2}{|c|}{ Primer trimestre } \\
\hline Porcentaje de peso para la talla (Rosso-M) & 61,0 \\
\hline Índice de masa corporal (Rosso-M y col.) & 60,0 \\
\hline Índice de masa corporal (Atalah y col.) & 64,0 \\
\hline IMCP (IME) & 72,0 \\
\hline Ganancia de peso (CLAP) & 69,0 \\
\hline \multicolumn{2}{|c|}{ Segundo trimestre } \\
\hline$\%$ peso para la talla (Rosso-M) & 63,0 \\
\hline Índice de masa corporal (Rosso-M y col.) & 63,0 \\
\hline Índice de masa corporal (Atalah y col.) & 69,0 \\
\hline Gradiente de peso materno según IMCP (IME) & 43,0 \\
\hline Ganancia de peso (CLAP) & 51,0 \\
\hline \multicolumn{2}{|c|}{ Tercer trimestre } \\
\hline \% peso para la talla (Rosso-M) & 59,0 \\
\hline Índice de masa corporal (Rosso-M y col.) & 56,0 \\
\hline Índice de masa corporal (Atalah y col.) & 70,0 \\
\hline Gradiente de peso materno según IMCP (IME) & 48,0 \\
\hline Ganancia de peso (CLAP) & 61,0 \\
\hline
\end{tabular}

IMCP: índice de masa corporal pregestacional

IME: Instituto de Medicina de los Estados Unidos

CLAP: Centro Latinoamericano de Perinatología y Desarrollo Humano 
5,7\% (cuadro 6). Los valores predictivos positivo y negativo incrementaron con todas las referencias antropométricas, excepto con la del Instituto de Medicina de Estados Unidos que perdió 12,8\% en el VPP; el más alto incremento del VPP se alcanzó con la referencia de Atalah y col. (14,3\%) y el más alto en VPN se presentó con la referencia del Centro Latinoamericano de Perinatología y Desarrollo Humano (6,3\%) (cuadro 6).

\section{Concordancia entre las referencias antropo- métricas al evaluar el estado nutricional de las gestantes}

Las más altas concordancias encontradas favorecieron a las tres referencias chilenas durante los tres controles gestacionales; la menor concordancia se observó con la referencia del Centro Latinoamericano de Perinatología y Desarrollo Humano. No obstante, en todos los casos la concordancia con esta última aumentó al final del embarazo sin cambio de categoría según el índice kappa (cuadro 7).

El porcentaje de peso para la talla de Rosso Mardones, con las demás referencias chilenas, tuvo una concordancia entre muy buena y moderada. El IMC de Rosso-Mardones concordó en la clasificación antropométrica con el IMC de Atalah y col. y fue entre buena y moderada. La concordancia con la referencia del Centro

Cuadro 6. Sensibilidad, especificidad y valores predictivos positivo y negativo de las referencias antropométricas gestacionales en el cada trimestre.

\begin{tabular}{|c|c|c|c|c|c|c|}
\hline \multirow[t]{2}{*}{ Muestra } & \multirow[b]{2}{*}{$\begin{array}{l}\text { Propiedades } \\
\text { métricas }\end{array}$} & \multicolumn{5}{|c|}{ Referencia antropométrica } \\
\hline & & $\begin{array}{c}\% \mathrm{P} / \mathrm{T} \\
\text { Rosso-M }\end{array}$ & $\begin{array}{c}\text { IMC } \\
\text { Rosso-M y col. }\end{array}$ & $\begin{array}{c}\text { IMC } \\
\text { Atalah y col }\end{array}$ & $\begin{array}{c}\text { Ganancia de peso } \\
\text { CLAP }\end{array}$ & $\begin{array}{l}\text { IMCP } \\
\text { IME }\end{array}$ \\
\hline & $\begin{array}{l}\text { Sensibilidad } \\
\text { (IC 95\%) }\end{array}$ & $\begin{array}{c}30,4 \% \\
(19,2-44,3)\end{array}$ & $\begin{array}{c}23,2 \% \\
(13,4-36,7)\end{array}$ & $\begin{array}{c}19,6 \% \\
(10,7-32,8)\end{array}$ & $\begin{array}{c}2,9 \% \\
(0,2-17,1)\end{array}$ & $\begin{array}{c}12,1 \% \\
(5,7-23,0)\end{array}$ \\
\hline \multirow[t]{4}{*}{$\begin{array}{l}\text { Primer } \\
\text { trimestre }\end{array}$} & $\begin{array}{l}\text { Especificidad } \\
\text { (IC 95\%) }\end{array}$ & $\begin{array}{c}72,0 \% \\
(64,2-78,7)\end{array}$ & $\begin{array}{c}73,2 \% \\
(65,5-79,8)\end{array}$ & $\begin{array}{c}80,3 \% \\
(73,0-86,0)\end{array}$ & $\begin{array}{c}97,4 \% \\
(90,2-99,6)\end{array}$ & $\begin{array}{c}93,9 \% \\
(89,0-96,7)\end{array}$ \\
\hline & $\begin{array}{l}\text { Valor predictivo + } \\
\text { (IC 95\%) }\end{array}$ & $\begin{array}{c}27,9 \% \\
(17,5-41,0)\end{array}$ & $\begin{array}{c}23,6 \% \\
(13,7-37,3)\end{array}$ & $\begin{array}{c}26,2 \% \\
(14,4-42,3)\end{array}$ & $\begin{array}{c}33,3 \% \\
(1,8-87,5)\end{array}$ & $\begin{array}{c}42,1 \% \\
(21,1-66,0)\end{array}$ \\
\hline & $\begin{array}{l}\text { Valor predictivo - } \\
\text { (IC 95\%) }\end{array}$ & $\begin{array}{c}74,3 \% \\
(66,5-80,9)\end{array}$ & $\begin{array}{c}72,8 \% \\
(65,0-79,4)\end{array}$ & $\begin{array}{c}73,7 \% \\
(66,3-80,0)\end{array}$ & $\begin{array}{c}69,7 \% \\
(60,1-78,0)\end{array}$ & $\begin{array}{c}74,3 \% \\
(68,0-79,8)\end{array}$ \\
\hline & $\begin{array}{l}\text { Sensibilidad } \\
\text { (IC 95\%) }\end{array}$ & $\begin{array}{c}28,8 \% \\
(18,6-41,4)\end{array}$ & $\begin{array}{c}30,3 \% \\
(19,9-43,0)\end{array}$ & $\begin{array}{c}19,7 \% \\
(11,3-31,7)\end{array}$ & $\begin{array}{c}56,1 \% \\
(43,3-68,1)\end{array}$ & $\begin{array}{c}66,6 \% \\
(53,9-77,5)\end{array}$ \\
\hline \multirow[t]{4}{*}{$\begin{array}{l}\text { Segundo } \\
\text { trimestre }\end{array}$} & $\begin{array}{l}\text { Especificidad } \\
\text { (IC 95\%) }\end{array}$ & $\begin{array}{c}76,0 \% \\
(68,9-81,9)\end{array}$ & $\begin{array}{c}74,9 \% \\
(67,7-80,9)\end{array}$ & $\begin{array}{c}87,2 \% \\
(81,1-91,5)\end{array}$ & $\begin{array}{c}49,2 \% \\
(41,7-56,7)\end{array}$ & $\begin{array}{c}34,1 \% \\
(27,3-41,6)\end{array}$ \\
\hline & $\begin{array}{l}\text { Valor predictivo + } \\
\text { (IC 95\%) }\end{array}$ & $\begin{array}{c}30,6 \% \\
(19,9-43,8)\end{array}$ & $\begin{array}{c}30,8 \% \\
(20,2-43,6)\end{array}$ & $\begin{array}{c}36,1 \% \\
(21,3-53,8)\end{array}$ & $\begin{array}{c}28,9 \% \\
(21,4-37,7)\end{array}$ & $\begin{array}{c}27,2 \% \\
20,6-34,8)\end{array}$ \\
\hline & $\begin{array}{l}\text { Valor predictivo - } \\
\text { (IC 95\%) }\end{array}$ & $\begin{array}{c}74,3 \% \\
(67,2-80,3)\end{array}$ & $\begin{array}{c}74,4 \% \\
(67,3-80,5)\end{array}$ & $\begin{array}{c}74,6 \% \\
(68,1-80,3)\end{array}$ & $\begin{array}{c}75,2 \% \\
(66,2-82,5)\end{array}$ & $\begin{array}{c}73,5 \% \\
(62,5-82,3)\end{array}$ \\
\hline & $\begin{array}{l}\text { Sensibilidad } \\
\text { (IC 95\%) }\end{array}$ & $\begin{array}{c}40,9 \% \\
(29,2-53,7)\end{array}$ & $\begin{array}{c}40,9 \% \\
(29,2-53,7)\end{array}$ & $\begin{array}{c}25,8 \% \\
(16,1-38,2)\end{array}$ & $\begin{array}{c}33,3 \% \\
(22,5-46,1)\end{array}$ & $\begin{array}{c}66,7 \% \\
(53,9-77,5)\end{array}$ \\
\hline \multirow[t]{3}{*}{$\begin{array}{l}\text { Tercer } \\
\text { trimestre }\end{array}$} & $\begin{array}{l}\text { Especificidad } \\
\text { (IC 95\%) }\end{array}$ & $\begin{array}{c}65,4 \% \\
(57,8-72,2)\end{array}$ & $\begin{array}{c}62,0 \% \\
(54,4-69,1)\end{array}$ & $\begin{array}{c}86,0 \% \\
(79,9-90,6)\end{array}$ & $\begin{array}{c}77,7 \% \\
(70,7-83,4)\end{array}$ & $\begin{array}{c}40,8 \% \\
(33,6-48,4)\end{array}$ \\
\hline & $\begin{array}{l}\text { Valor predictivo + } \\
\text { (IC 95\%) }\end{array}$ & $\begin{array}{c}30,3 \% \\
(21,3-41,1)\end{array}$ & $\begin{array}{c}28,4 \% \\
(19,9-38,7)\end{array}$ & $\begin{array}{c}40,5 \% \\
(26,0-56,7)\end{array}$ & $\begin{array}{c}35,5 \% \\
(24,0-48,7)\end{array}$ & $\begin{array}{c}29,3 \% \\
(22,3-37,4)\end{array}$ \\
\hline & $\begin{array}{l}\text { Valor predictivo - } \\
\text { (IC 95\%) }\end{array}$ & $\begin{array}{c}75,0 \% \\
(67,3-81,4)\end{array}$ & $\begin{array}{c}74,0 \% \\
(66,1-80,7)\end{array}$ & $\begin{array}{c}75,9 \% \\
(69,3-81,5)\end{array}$ & $\begin{array}{c}76,0 \% \\
(69,0-81,8)\end{array}$ & $\begin{array}{c}76,8 \% \\
(66,8-84,6)\end{array}$ \\
\hline
\end{tabular}

IMCP: índice de masa corporal pregestacional

IME: Instituto de Medicina de los Estados Unidos

CLAP: Centro Latinoamericano de Perinatología y Desarrollo Humano 
Cuadro 7. Concordancia entre las referencias utilizadas en la clasificación antropométrica del estado nutricional de las gestantes según trimestre.

\begin{tabular}{|c|c|c|}
\hline \multicolumn{2}{|l|}{ Referencias antropométricas } & \multirow[t]{2}{*}{ Índice Kappa } \\
\hline & Primer trimestre & \\
\hline \%P/T Rosso-M vs IMC Rosso-M y col. & & $\begin{array}{l}0,887 \\
\text { (ES: } 0,026 ; \text { IC } 95 \%: 0,836-0,938)\end{array}$ \\
\hline IMC Rosso-M y col. vs IMC Atalah y col. & & $\begin{array}{c}0,670 \\
\text { (ES: } 0,039 ; \text { IC } 95 \%: 0,594-0,746)\end{array}$ \\
\hline \%P/T Rosso-M vs IMC Atalah y col. & & $\begin{array}{c}0,614 \\
\text { (ES: } 0,041 ; \text { IC } 95 \%: 0,534-0,694)\end{array}$ \\
\hline \%P/T Rosso-M vs CLAP & & $\begin{array}{c}0,006 \\
\text { (ES: } 0,031 ; \text { IC } 95 \%-0,055-0,067)\end{array}$ \\
\hline IMC Rosso-M y col. vs CLAP & & $\begin{array}{c}-0,009 \\
\text { (ES: } 0,031 ; \text { IC } 95 \%:-0,070-0,052)\end{array}$ \\
\hline \multirow[t]{2}{*}{ IMC Atalah y col. vs CLAP } & & (ES: 0,$035 ;$ IC 95\%: $-0,067-0,071)$ \\
\hline & Segundo trimestre & \\
\hline \%P/T Rosso-M vs IMC Rosso-M y col. & & $\begin{array}{c}0,871 \\
\text { (ES: } 0,026 ; \text { IC 95\%: } 0,820-0,922)\end{array}$ \\
\hline \%P/T Rosso-M vs IMC Atalah y col. & & (ES: 0,$039 ;$ IC 95\%: $0,519-0,671)$ \\
\hline IMC Rosso- M y col. vs IMC Atalah y col. & & $\begin{array}{l}0,561 \\
\text { (ES: } 0,038 \text {; IC 95\%: } 0,487-0,635)\end{array}$ \\
\hline \%P/T Rosso-M vs CLAP & & $\begin{array}{c}-0,001 \\
\text { (ES: } 0,037 ; \text { IC } 95 \%:-0,073-0,071)\end{array}$ \\
\hline IMC Rosso- M y col. vs CLAP & & $\begin{array}{c}0,024 \\
\text { (ES: } 0,037 ; \text { IC 95\%: }-0,048-0,096)\end{array}$ \\
\hline \multirow[t]{2}{*}{ IMC Atalah y col. vs CLAP } & & $\begin{array}{l}0,061 \\
\text { (ES: } 0,035 ; \text { IC } 95 \%:-0,008-0,129)\end{array}$ \\
\hline & Tercer trimestre & \\
\hline \%P/T Rosso-M vs IMC Atalah y col. & & $\begin{array}{c}0,539 \\
\text { (ES: 0,040; IC 95\%: 0,461-0,617) }\end{array}$ \\
\hline \%P/T Rosso-M vs IMC Rosso y col. & & $\begin{array}{c}0,758 \\
\text { (ES: } 0,032 ; \text { IC 95\%: } 0,695-0,820)\end{array}$ \\
\hline IMC Rosso- M y col. vs IMC Atalah y col. & & $\begin{array}{l}0,497 \\
\text { (ES: } 0,036 ; \text { IC } 95 \%: 0,426-0,568)\end{array}$ \\
\hline IMC Rosso- M y col. vs CLAP & & $\begin{array}{l}0,112 \\
\text { (ES: } 0,030 ; \text { IC } 95 \%: 0,037-0,186)\end{array}$ \\
\hline IMC Atalah y col. vs CLAP & & $\begin{array}{l}0,067 \\
\text { (ES: } 0,043 ; \text { IC } 95 \%:-0,017-0,151)\end{array}$ \\
\hline \%P/T Rosso-M vs CLAP & & $\begin{aligned} & 0,117 \\
\text { (ES: } 0,042 ; & \text { IC } 95 \%: 0,035-0,199)\end{aligned}$ \\
\hline
\end{tabular}

Índice Kappa: pobre:<0,20; regular: 0,21 - 0,40; moderada: 0,41 - 0,60; buena: 0,61 - 0,80; muy buena: 0,81 - 1,00 (Altman DG 1991).

ES: error estándar

IMCP: índice de masa corporal pregestacional

IME: Instituto de Medicina de los Estados Unidos

CLAP: Centro Latinoamericano de Perinatología y Desarrollo Humano

Latinoamericano de Perinatología y Desarrollo Humano fue baja en todos los trimestres y con todas las referencias antropométricas. Con la referencia del Instituto de Medicina de Estados Unidos sólo se pudo calcular la concordancia en el primer trimestre porque se encontraron casillas vacías o con muy pocos datos en la categoría de normal en el segundo y tercer trimestres, razón por la que no se incluyó en el cuadro (cuadro 7).

\section{Discusión}

En Colombia el avance en la vigilancia nutricional de la embarazada y en el control del peso insuficiente al nacer $(<3.000 \mathrm{~g})$ relacionado con la antropometría 
materna es muy incipiente, lo que se constata en la flexibilidad del Ministerio de la Protección Social cuando utiliza cualquiera de las referencias antropométricas en grupos de diverso origen étnico y con puntos de corte diferentes (33). Con excepción de Chile, en la mayoría de los países de América Latina la situación es similar; se ha alcanzado mayor madurez en la vigilancia nutricional del menor de cinco años que de la gestante.

Además de la falta de orientación clara sobre cuál referencia antropométrica gestacional es la más adecuada para nuestro contexto, la autonomía que otorgó la Ley 100 de Seguridad Social en Salud a las instituciones del sector dificulta la comparación regional y nacional de la prevalencia de malnutrición de las gestantes colombianas, así como la definición de una política pública de nutrición materna.

Vigilar el estado nutricional de la embarazada, o tamizarla en la primera consulta para remitirla a un nivel superior de atención en salud, requiere contar con un instrumento de buena capacidad de predicción del peso al nacer. Los estudios realizados en Colombia concuerdan con éste en cuanto a la baja capacidad predictiva (sensibilidad, especificidad y valores predictivos) del peso al nacer de las referencias del Centro Latinoamericano de Perinatología y Desarrollo Humano $(34,37)$ y de Rosso-Mardones $(35,37)$. No se ha publicado hasta la fecha un estudio que haya incluido todas las referencias gestacionales disponibles en el país y estudiadas aquí.

Los autores de las cinco referencias evaluadas consideraron similares criterios de inclusión y exclusión en su diseño; sin embargo, todas las referencias fueron elaboradas con poblaciones de diferentes características raciales y antropométricas, niveles socioeconómicos y objetivos programáticos, lo que explicaría parcialmente la baja capacidad predictiva encontrada. No obstante, los criterios para la clasificación inicial de la gestante y la meta proyectada de ganancia de peso fueron los que más separaron la referencia del Centro Latinoamericano de Perinatología y Desarrollo Humano y la del Instituto de Medicina de Estados Unidos de las tres referencias gestacionales chilenas.
Las expectativas muy altas frente a la ganancia de peso o al peso inicial gestacional pudieron llevar a incrementar el total de falsos positivos o de madres clasificadas con bajo peso gestacional sin una correspondencia con el peso al nacer del bebé. Esto fue evidente en cada trimestre al observar valores menores de exactitud diagnóstica con las referencias que arrojaron las mayores proporciones de bajo peso gestacional.

Un procedimiento o referencia de identificación de sujetos en riesgo consta usualmente de una variable separadora o discriminante (37) y de un punto de corte. En la práctica, ningún procedimiento es capaz de lograr una separación perfecta, por lo que siempre aparecerán casos mal clasificados. Es lógico pensar que la mejor referencia antropométrica gestacional es aquélla que consigue identificar correctamente la mayor proporción de madres en riesgo; sin embargo, se sabe que esta proporción no depende sólo de las propiedades métricas de la referencia, sino también de la prevalencia del evento cuyo riesgo se desea detectar $(10,13)$.

Las referencias antropométricas gestacionales de más disímil comportamiento en este estudio fueron la del Instituto de Medicina de los Estados Unidos y la del Centro Latinoamericano de Desarrollo Humano y Perinatología. Se diferenciaron en que la propuesta de la de Estados Unidos correspondió a una tasa o gradiente de ganancia de peso esperada de acuerdo con el IMC pregestacional y la del Centro Latinoamericano, a un valor absoluto total a partir de un peso previo teórico. También se diferenciaron en que la referencia del Instituto de Medicina de los Estados Unidos planteó tasas diferenciales de ganancia de peso por categorías de IMC pregestacional y la del Centro Latinoamericano de Desarrollo Humano y Perinatología no discriminó la ganancia total según el estado nutricional inicial de la madre.

El origen del peso teórico de la referencia del Centro Latinoamericano de Desarrollo Humano la separó aún más de las otras referencias antropométricas, pues para aquellas embarazadas que desconocían su peso pregestacional, situación frecuente en nuestros países, estableció un punto de corte inicial muy alto, que no tuvo 
correspondencia con la ganancia de peso a partir del segundo trimestre. Esta situación fue evidente en el alto porcentaje de madres clasificadas con sobrepeso en el primer trimestre, el cual disminuyó abruptamente a partir de allí, y también en el incremento notable de la proporción de bajo peso gestacional a partir del segundo trimestre.

Con estas dos referencias antropométricas se presentaron las más bajas proporciones de bajo peso gestacional en el primer trimestre, al igual que las mayores especificidades y exactitud diagnóstica; no obstante, al final del embarazo presentaron sensibilidades muy superiores, pues las madres no lograron la meta de ganancia de peso propuesta por los autores (Instituto de Medicina de los Estados Unidos y Centro Latinoamericano de Desarrollo Humano y Perinatología).

Según Habicht (10), la sensibilidad y la especificidad para un tipo de desnutrición pueden ser estables entre una y otra población; no obstante, estos dos conceptos exhiben una relación inversa: para una variable separadora dada, el punto de corte determina el valor de la sensibilidad y la especificidad. Incrementar o disminuir el punto de corte tiene un efecto diferente sobre la sensibilidad y la especificidad. Su selección se subordina entonces a la capacidad de intervención y puede manipularse arbitrariamente, de suerte que el número de beneficiarias sea igual al número de intervenciones posible.

Si se parte de la premisa anterior, las referencias gestacionales del Instituto de Medicina de los Estados Unidos y la del Centro Latinoamericano de Desarrollo Humano y Perinatología dejarían por fuera a un importante número de gestantes que debería ser tamizado para intervención nutricional en el primer trimestre con el propósito de proteger al feto de resultados adversos al nacer.

Por otro lado, la concordancia observada en la clasificación antropométrica gestacional entre las tres referencias chilenas podría servir como argumento para usar cualquiera de ellas en la evaluación antropométrica de la gestante; sin embargo, a pesar de que en los dos primeros trimestres se comportaron de manera similar en la clasificación del bajo peso gestacional, no fue así en la clasificación de obesidad y de sobrepeso gestacional, lo que confirma los hallazgos de Erazo y col. en el 2004 (32). Con la transición nutricional que se observa en Colombia se debe tener en cuenta la clasificación de sobrepeso y obesidad y estudiar los incrementos de peso deseados en estos casos para proteger al binomio madre - hijo de resultados adversos.

Si el objetivo de un sistema de vigilancia nutricional fuera la detección precoz (primer trimestre) de las embarazadas con mayor proporción de bajo peso se impondría la elección de las referencias de Rosso-Mardones; de igual manera, la selección favorecería a las referencias de Rosso-Mardones si el objetivo fuera la detección del sobrepeso o de la obesidad gestacional.

Los procedimientos de pesquisa no pueden extrapolarse de una población a otra, particularmente en lo que concierne a la elección de los puntos de corte. Esta consideración es esencial a efectos de elegir una referencia antropométrica con fines de tamizaje $(10,11,15,37)$.

Sí la distribución de los factores que afectan el peso al nacer es diferente entre dos poblaciones, la sensibilidad y la especificidad de un indicador con respecto al mismo punto de corte pueden también cambiar (11), tal como se encontró en este estudio y en los realizados en Medellín, 19911992 (33), Bogotá, 1994 (34), Cuba, 1995 (35), Manizales, 1996-1997 (36) y Chile, 1997 (37) y 2004 (32). Vale la pena resaltar que la sensibilidad calculada en todos los estudios no superó el $60,0 \%$, lo cual permitió confirmar el bajo poder predictivo de la antropometría materna para el peso al nacer (32), aun con la aplicación de todos los criterios de inclusión que se tuvieron en cuenta.

De acuerdo con lo expuesto, existen diferentes criterios para seleccionar una referencia antropométrica de tamizaje de las embarazadas colombianas en riesgo de tener hijos con peso insuficiente al nacer $(10,11,15,37)$. La elección tiene implicaciones económicas para el sistema de salud y de salud y calidad de vida para el binomio madre-niño y dependerá del uso de las referencias en clínica o en salud pública. 
Con el propósito de lograr un impacto oportuno y pertinente en la madre y en el feto se debería tamizar a la embarazada entre el primer y segundo trimestres. En consecuencia, al elegir una referencia de acuerdo con su mayor especificidad (primer trimestre, la del Centro Latinoamericano de Desarrollo Humano y Perinatología; segundo trimestre, la de Atalah y col.), se sacrificaría su sensibilidad; en este caso, el sistema de vigilancia nutricional protegería a la madre de intervenciones nutricionales innecesarias (55).

Si por el contrario se escogiera la referencia antropométrica de acuerdo con su mayor sensibilidad (primer trimestre, el \% P/T de Rosso-Mardones; segundo trimestre, la del Instituto de Medicina de los Estados Unidos), se estaría privilegiando el estado nutricional del feto y debería incluirse como objetivo del sistema de vigilancia la atención nutricional de la madre después del parto para recuperar y mantener un adecuado estado nutricional y protegerla del riesgo de enfermedades crónicas por mantener el peso posparto en las gestantes falsas positivas (55).

Si se quisiera intervenir a las gestantes que realmente se beneficiarían con la intervención nutricional en comparación con las que la reciben (en función de la prevalencia), se tendría que considerar la referencia con el valor predictivo positivo más alto, en este caso la del Instituto de Medicina de los Estados Unidos en el primer trimestre o la de Atalah y col. en el segundo trimestre. Por lo tanto, aun cuando se simplifiquen los supuestos asociados a los costos y a los efectos adversos de la intervención, el mejor nivel para el tamizaje no sólo depende de la sensibilidad y la especificidad, sino también de la prevalencia del peso insuficiente al nacer $(15,56)$.

Así pues, lo más aconsejable sería seleccionar la referencia antropométrica de acuerdo con su mayor exactitud diagnóstica porque incluye tanto la sensibilidad como la especificidad y elimina al máximo los falsos positivos y negativos. Esto llevaría a escoger la referencia de Atalah y col. con el apoyo de una estrategia adecuada para su aplicación precoz en el primer trimestre del embarazo.
Debido a la aplicación estricta de los criterios de inclusión y exclusión que se tuvieron en cuenta en este estudio, se recomienda aplicar la referencia de Atalah y col. en poblaciones de diversas características de salud y antecedentes obstétricos con el propósito de estimar su exactitud diagnóstica y confirmar o no sus cualidades métricas para predecir el peso insuficiente al nacer.

\section{Agradecimientos}

Expreso mis agradecimientos a la Universidad de Caldas por su apoyo financiero y por el tiempo que me concedió para dedicarlo a mis estudios. A ASSBASALUD por permitirme recolectar la información en su programa de Control Prenatal. A Jorge Bacallao $G$ por sus valiosos aportes como tutor, a Rafael Jiménez por su asesoría en el diseño del estudio y a Pedro Monterrey por su excelente orientación metodológica. Por último, agradezco a las mujeres gestantes que voluntariamente participaron en el desarrollo de este proyecto y permitieron su materialización.

\section{Conflicto de intereses}

Participé en este proyecto como única investigadora con la asesoría de Jorge Bacallao y Rafael Jiménez del Instituto Superior de Ciencias Médicas de La Habana, Cuba.

\section{Financiación}

Este proyecto contó con apoyo financiero de la Universidad de Caldas (Oficio 1185 de marzo 14/01 de la Vicerrectoría de Investigaciones y Postgrados de la Universidad de Caldas) y de la autora como estudiante de doctorado en Ciencias de la Salud.

\section{Referencias}

1. Abrams B, Parker JD. Maternal weight gain in women with good pregnancy outcome. Obstet Gynecol 1990;76:1-7.

2. World Health Organization. Maternal anthropometry and pregnancy outcomes. A WHO collaborative study. Bull World Health Organ 1995;73(Suppl):1-98.

3. Abrams B, Altman SL, Pickett KE. Pregnancy weight gain: still controversial. Am J Clin Nutr 2000;71(5 Suppl):1233S-41S.

4. Murakami M, Ohmichi M, Takahashi T, Shibata A, Fukao A, Morisaki N et al. Prepregnancy body mass 
index as an important predictor of perinatal outcomes in Japanese. Arch Gynecol Obstet 2005;271:311-5.

5. Moore VM, Davies MJ. Diet during pregnancy, neonatal outcomes and later health. Reprod Fertil Dev 2005; $17: 341-8$

6. Hediger ML, Luke B, van de Ven C, Nugent C. Midupper arm circumference (MUAC) changes in late pregnancy predict fetal growth in twins. Twin Res Hum Genet 2005;8:267-70.

7. Loos RJ, Derom C, Derom R, Vlietinck R. Determinants of birthweight and intrauterine growth in liveborn twins. Paediatr Perinat Epidemiol 2005;19(Suppl 1): $15-22$.

8. Kulvanitchaiyanunt A. Study of the prognostic value of the pregnant nutrition graph (Vallop Curve) to predict the incidence of low birth weight infants. J Med Assoc Thai 2005;88:9-14

9. Kac G, Velásquez-Meléndez G. Gestational weight gain and macrosomia in a cohort of mothers and their children. J Pediatr (Rio J) 2005;81:47-53.

10. Habicht JP. Some characteristics of indicators of nutritional status for use in screening and surveillance. Am J Clin Nutr 1980;33:531-5.

11. Habicht JP, Meyers LD, Brownie C. Indicators for identifying and counting the improperly nourished. Am J Clin Nutr 1982;35(5 Suppl):1241-54.

12. Brownie C, Habicht JP. Selecting a screening cut-off point or diagnostic criterion for comparing prevalences of disease. Biometrics 1984;40:675-84.

13. Brownie C, Habicht JP, Cogill B. Comparing indicators of health or nutritional status. Am J Epidemiol 1986;124:1031-44

14. Habicht JP, Pelletier DL. The importance of context in choosing nutritional indicators. J Nutr 1990;120(Suppl 11): 1519-24.

15. Habicht JP, Stoltzfus RJ. What do indicators indicate? Am J Clin Nutr 1997;66:190-1.

16. Habicht JP. Comparing the quality of indicators of nutritional status by receiver operating characteristic analysis or by standardized differences. Am J Clin Nutr 2000;71:672-3.

17. Dorlencourt F, Priem V, Legros D. Anthropometric indices used for the diagnosis of malnutrition in adolescents and adults: review of the literature. Bull Soc Pathol Exot 2000;93:321-4

18. de Paoli I, Sánchez A, Pérez G. La efectividad de los indicadores antropométricos en el diagnóstico nutricional de mujeres embarazadas bien y desnutridas. Arch Latinoam Nutr 2001;51:346-50.

19. Krasovec K, Anderson MA. Maternal nutrition and pregnancy outcomes. Anthropometric assessment.
First ed. (Scientific Publication No. 529). Washington DC: United States Agency for International Development, Mother Care, Pan American Health Organization, World Health Organization; 1991. p.214.

20. Gueri M, Jutsum P, Sorhaindo B. Anthropometric assessment of nutritional status in pregnant women: a reference table of weight-for-height by week of pregnancy. Am J Clin Nutr 1982;35:609-16.

21. Rosso P. A new chart to monitor weight gain during pregnancy. Am J Clin Nutr 1985;41:644-52.

22. Institute of Medicine (U.S.), Subcommittee on Nutritional Status and Weight Gain during Pregnancy, Institute of Medicine (U.S.), Subcommittee on Dietary Intake and Nutrient Supplements during Pregnancy. Nutrition during pregnancy: part I, weight gain, part II, nutrient supplements. Washington, D.C.: National Academy Press; 1990.

23. Organización Mundial de la Salud. Mujeres embarazadas y mujeres lactantes. En: Informe de un Comité de expertos de la OMS. El estado físico: uso e interpretación de la antropometría. Ginebra: Organización Mundial de la Salud; 1995. p.45-139.

24. de Siqueira AA, Tanaka AC, Junior CC, de Almeida PA. The use of a normal pregnant women's weight curve in the diagnosis of intrauterine malnutrition. Rev Saude Publica 1975;9:495-506.

25. de Siqueira AA, Ciari Jr C, Mattos IL, Buralli KO, Baptista Filho M, Schor $\mathbf{N}$ et al. Application of a weight gain curve for pregnant women. Rev Saude Publica 1977;11:288-93

26. Fescina RH. Aumento de peso durante el embarazo. Método para su cálculo cuando se desconoce el peso habitual. Bol Of Sanit Panam 1983;95:156-61.

27. Schwarcs R, Díaz AG, Fescina RH, De Mucio B, Belitzky R, Delgado L. Salud reproductiva materna perinatal. Atención prenatal y del parto de bajo riesgo. (Publicación Científica No 1321.01). Montevideo: Centro Latinoamericano de Perinatología y Desarrollo Humano; 1995.

28. Mardones-Santander F, Rosso P. Design of a weight gain chart for pregnant women. Rev Med Chile 1997;125:1437-48.

29. Mardones-Santander F, Rosso P, Marshall G, Villaroel I, Bastías G. Comparación de los indicadores de la relación peso-talla en la embarazada. Acta Pediatr Esp 1999;57:573-8.

30. Atalah E, Castillo C, Gómez C, Mateluna A, Urteaga C, Castro $\mathbf{R}$ et al. Malnutrition of the pregnant woman: an overestimated problem? Rev Med Chil 1995; 123:1531-8.

31. Atalah E, Castillo C, Castro R, Aldea A. Proposal of a new standard for the nutritional assessment of pregnant women. Rev Med Chil 1997;125:1429-36. 
32. Erazo M, Lagos R. Estado nutricional materno y determinación de un instrumento para su evaluación. Informe Técnico. Santiago de Chile: Universidad de Chile - Universidad de La Frontera -Hospital Regional de Temuco; 2004.

33. Martínez L. Validación de instrumentos para monitorear la ganancia de peso gestacional. Revista Nutrición y Dietética 1994;2:18-26.

34. Fundación Santa Fe de Bogotá. Gráfica de incremento de peso para embarazadas. Prueba para Bogotá de la gráfica de Rosso-Mardones. Estudio colaborativo. Informe final. Bogotá: Fundación Santa Fé de Bogotá; 1995. p.1-21.

35. Jiménez R, Bacallao J. Prognostic performance of several anthropometrics indicators for predicting low and insufficient birth weight. Am J Hum Biol 1995;7:303-11.

36. Benjumea MV, Muñoz L, Ríos A. Evaluación de la capacidad predictiva de las curvas de RossoMardones y del CLAP para bajo peso al nacer. Informe Técnico. Manizales: Universidad de Caldas, Universidad Católica de Manizales, Secretaría de Salud de Manizales; 1997.

37. Bacallao J, Jiménez R. Análisis comparativo de dos criterios de referencia para tamizaje nutricional de la embarazada. Rev Chil Nutr 1999;26:223-32.

38. Dirección General de Promoción y Prevención Ministerio de Salud, República de Colombia. Norma técnica para la detección temprana de las alteraciones del embarazo. Resolución 412. Bogotá: República de Colombia. Ministerio de Salud; 2000. p.1-17.

39. Departamento Administrativo Nacional de Estadística. Información estadística. Nacidos vivos por peso al nacer 2004. Cuadro 8. [Consultado: agosto 20 de 2006]. Disponible en: http://www.dane.gov.co/files/ investigaciones/poblacion/nacimientos/ nacimientos 2004/Cuadro8.xls.

40. Arango F. Base de datos de peso al nacer y morbilidad en el servicio de neonatología. Manizales: Hospital de Caldas, Universidad de Caldas; 1998-2004.

41. Puffer R, Serrano C. Características del peso al nacer. (Publicación Científica No. 504.) Washington DC: Organización Panamericana de la Salud; 1988. p.112.

42. Carroli G, Villar J, Piaggio G, Khan-Neelofur D, Gulmezoglu M, Mugford M et al. WHO systematic review of randomised controlled trials of routine antenatal care. Lancet 2001;357:1565-70.

43. Szostak-Wegierek D, Szamotulska K, Szponar L. Influence of maternal nutrition on infant birthweight. Ginekol Pol 2004;75:692-8.

44. Wen SW, Walker M. An exploration of health effects of folic acid in pregnancy beyond reducing neural tube defects. J Obstet Gynaecol Can 2005;27:13-9.
45. Carabin H, Cowan LD, Beebe LA, Skaggs VJ, Thompson D, Agbangla C. Does participation in a nurse visitation programme reduce the frequency of adverse perinatal outcomes in first-time mothers? Paediatr Perinat Epidemiol 2005;19:194-205.

46. Hosain GM, Chatterjee N, Begum A, Saha SC. Factors Associated with Low Birthweight in Rural Bangladesh. J Trop Pediatr. 2006;52:87-91.

47. Lohman TG, Roche A, Martorell R. Anthropometric standardization reference manual. Primera ed. Champaign, Illinois: Human Kinetics Books; 1988.

48. Benjumea MV. Bioética en la vigilancia epidemiológica de la gestante colombiana. Revista Perspectivas en Nutrición Humana 2003;9:46-71.

49. Ministerio de Salud, República de Colombia. Resolución Número 008430. Bogotá: República de Colombia. Ministerio de Salud; Octubre 4 de 1993. p.1-12.

50. Fescina RH, Quevedo C, Martell M, Nieto F, Schwarcs R. Altura uterina como método para predecir el crecimiento fetal. Bol Ofic Sanit Panam 1984 96:377-86.

51. Fescina RH, Schwarcsz R, Díaz AG. Vigilancia del crecimiento fetal. Manual de autoinstrucción. (Publicación Científica CLAP No 1261). Montevideo: Organización Panamericana de la Salud; 1992. p.231.

52. Burrows R, Castillo C, Atalah E, Uauy R. Guías de alimentación para la mujer. Primera Edición. Santiago de Chile: Gobierno de Chile, Ministerio de Salud, Universidad de Chile; 2001.

53. Sánchez J, Santos A, Santamaría C, Benito C, Benito M, Martín A et al. Discriminación de tejidos cerebrales mediante espectroscopía por resonancia magnética de alta y baja resolución: Estudio comparativo. 1998. [Consultado: noviembre 15 de 2005]. Disponible en:http://www.die.upm.es/im/papers/J Scaseib. pdf\#search='funci\%C3\%B3n\%20 Discriminante \% 20 de\%20Fisher.

54. Riegelman RK, Hirsch RP. Cómo estudiar un estudio y probar una prueba: lectura crítica de la literatura médica. Segunda Edición. (Publicación Científica No 531). Washington DC: Organización Panamericana de la Salud; 1992. p.260

55. Altman DG. Practical statistics for medical research. First ed. London: Chapman \& Hall; 1991.p.140-1.

56. Kramer MS, Haas J, Kelly A. Maternal anthropometrybased screening and pregnancy outcome: a decision analysis. Trop Med Int Health 1998;3:447-53. 